\title{
A Contrastive Study between Machine Translation and Human Translation: Taking Japanese Translation Application as an Example
}

\author{
Guifang Zhang, Zixi Wei, Rui Zhao* \\ Southwest Minzu University, Chengdu, China \\ Email: *zgfsaikou@163.com
}

How to cite this paper: Zhang, G.F., Wei, Z.X. and Zhao, R. (2021) A Contrastive Study between Machine Translation and Human Translation: Taking Japanese Translation Application as an Example. Open Access Library Journal, 8: e7769.

https://doi.org/10.4236/oalib.1107769

Received: July 16, 2021

Accepted: August 1, 2021

Published: August 4, 2021

Copyright $\odot 2021$ by author(s) and Open Access Library Inc.

This work is licensed under the Creative Commons Attribution International License (CC BY 4.0).

http://creativecommons.org/licenses/by/4.0/

\begin{abstract}
With the development of artificial intelligence, machine translation is making progress. This paper will take Japanese translation application as an example to analyze the contrast differences between machine translation and human translation, and discuss the relationship between them and the prospect for the future.
\end{abstract}

\section{Subject Areas}

Linguistics

Keywords

Artificial Intelligence, Machine Translation, Human Translation, Contrast Difference, Relationship

\section{1. 引言}

近年来, 关于人工智能是否能取代人工这一类话题一直争论不休。随着 翻译类 APP 不断改进, 这类翻译机器会取代人工翻译吗? 如今翻译类 APP 被广泛应用。那么, 翻译出来的内容是否 “达意” ? 和人工翻译又有何区别? 本文将会比较机器翻译及人工翻译的差异, 试就对两者进行探讨与思考。

\section{2. 两种重要的机器翻译方式}

目前, 最重要的两种机器翻译方式是规则法和统计法。规则法简单来说 
就是由有限的规则推导出来。需要语言学家来写规则, 这一个词翻译成另外 一个词、这个成分翻译成另外一个成分、出现在句子的什么位置, 都用规则 表示出来。因此, 基于规则的系统开发周期很长, 成本很高。此外, 随着规 则数量的增多, 规则之间互相影响, 就会产生规则混乱问题。为了解决问题 而再写一个规则, 这样一来, 就不得不引入了更多的规则, 形成恶性循环。

统计法则是一个句子对应多个不同概率的句子译文, 最终找到概率最大 的句子。例如 “一起去散步吗” 可以译成 “散歩しましょうか” 或者 “一緒 に散歩しますか”, 经过训练的翻译机器就会分析两者的匹配概率, 前者概 率大于后者, 最终译文就会是 “散步しましょうか”。那么翻译机器如何进 行训练呢? 统计机器翻译系统对机器翻译进行了一个数学建模, 让它在大数 据的基础上进行自动训练, 并形成翻译知识。翻译知识主要来自两类训练数 据: 平行语料及单语语料。平行语料可以训练机器的翻译模型。比如 “吃” 可以翻译为 “食べる”, 而 “食べる” 后面会有一个概率来衡量两个词对应 的可能性。这样, 平行语料便建立了两种语言相联系的桥梁。单语语料可以 训练机器的语言模型, 衡量翻译的语言是否流利。比如 “ご飯を食べる”。 那么 “スープを食べる” 可以不可以呢? “食べる”与 “スープ” 搭配的概 率很低, 不符合语言表达, 机器不会这样翻译。统计机器翻译以数据为基础, 如果数据稀少, 就难以进行 “训练”。

不管是哪种方式, 其本质都是二度摹仿的数据处理, 具有机械化的特点。 只有人工输入数据之后, 翻译机器才可以通过数据进行翻译, 所以机器翻译 无法完全离开人工翻译。

\section{3. 机器翻译与人工翻译的内容比较}

有学者指出: “要提高机译的质量, 首先要解决的是语言本身问题而不 是程序设计问题; 单靠若干程序来做机译系统, 肯定是无法提高机译质量的; 另外在人类尚未明了大脑是如何进行语言的模糊识别和逻辑判断的情况下, 机译要想达到 “信、达、雅” 的程度是不可能的。” 那么, 国内外企业陆续 推出的翻译类 APP 的实际应用情况是怎样的呢? 接下来笔者将会把百度、网 易有道、腾讯、谷歌翻译 APP 的翻译内容与人工翻译的内容进行比较。

\section{1. 汉译日}

例 1 【原文】她哧哧地笑起来, 说: “这么个小东西, 也知道捉螃蟹?” (对 捉螃蟹的小孩说)

【人工译文】彼女はウフフと笑い出して言った。「こんなおちびさん でも、蟹の取り方しってるの? 」[1]

【百度译文】彼女はくすくす笑って言いました。「こんな小さいもの はカ二を捕まえることも知っていますか? 」

【网易有道译文】彼女はくすくす笑って、「こんな小さいものでも、 蟹を捕るのか」と言った。

【腾讯译文】彼女は食べながら笑って, “こんな小さなものでも, カ 二を捕るのを知っているのか?”と言った. 
【谷歌译文】彼女は笑って言った、「あなたはそのょうな小さなこと のためにカ二を捕まえる方法を知っていますか? 」

汉语中的 “东西”一词不完全等同于日语中的 “東西”，甚至，汉语的 “东西” 含义更广。现代汉语中, “东西” 除了泛指各种抽象的或具体的事 物之外, 还指人或动物(多含厌恶或喜爱的感情), 如 “蚟东西”。这里的 “小 东西” 是对小孩的昵称, 故译为 “おちびさん”。而前三个机器翻译都翻译 为 “もの”, “もの” 在日语中虽然也可以指人, 但是用 “小さなもの” 描 述人相当奇怪, 更像是指 “小物品”。谷歌翻译 “こと” 更不必说了。此外, 有的机器翻译存在标点符号错误问题。总的来说, 机器翻译能基本完成句子 译文转换, 但是在细节还是做的不够精准, 会造成误译。

例 2【原文】下了一场雨。

【人工译文】雨が降った。[2]

【百度译文】一雨降った。

【网易有道译文】雨が降った。

【腾讯译文】一雨降った。

【谷歌译文】雨が降っている。

在汉译日的翻译中, 句子中的 “一只、一场” 等一般不进行翻译。在日 语的表达中, 类似这样的数量词没有实际意义。如果说它们起到一定表达作 用的话, 就是表示单数。而从与日语比较的角度看, 在修饰主语时, 这样的 数量词与 “が” 相对应, 可省略。只有表示一定程度的强调时, 才必须翻译 出来。机器翻译中, 有的把数量词翻译出来了; 有的存在语态错误, “了” 是过去时态, 不能用 “ている”。此外, 百度翻译和腾讯翻译的相同译文表 明, 即使是不同的翻译机器, 也会有相同的运作规则。这是机器翻译的机械 性特点的体现。

\section{2. 日译汉}

例 3【原文】猫の額程な町内の癖に、中学校のありかも知ら奴があ るものか。

【人工译文】巴掌大的镇子, 竟然有人不知道中学校在什么地方! [3]

【百度译文】区里只有猫那么大的一块钱, 怎么会有人不知道中学有没 有呢。

【网易有道译文】像猫一样大小的街坊, 哪有连中学在哪里都不知道的 家伙。

【腾讯译文】像猫额头那么大的街道上, 有没有连中学都不知道的家伙 啊。

【谷歌译文】有没有人甚至不知道猫附近有中学的存在?

“猫の額” 是日语惯用语。虽然日语惯用语一般由两个以上的词语组成, 但并不意味着简单的相加, 有时甚至会与表意相差甚远。“猫の額” 表示像 猫的额头那样窄小, 即 “面积窄小”。如果直译就会闹出笑话。“ものか” 在词句中表达一种主观性的、感叹的语气, 在此可译为 “居然”、“竟然” 等, 腾讯翻译和谷歌翻译却译为 “有没有”, 显然没有读懂这句话的语境。 
例 4【原文】私はお天気屋だから、店員が不親切だったり、ブスッと した顔でいられるとカチンとくる。

【人工译文】我这个人很情绪化, 如果营业员态度冷淡或绷着脸, 就会 生气。[3]

【百度译文】因为我是天气店, 店员不亲切, 或者是一脸不整洁的样子 会让我生气。

【网易有道译文】我是个天气行家, 如果店员不热情, 或者一脸冷漠, 我就会生气。

【腾讯译文】因为我是个天气店, 所以店员不亲切, 或者一脸毛骨悚然 的样子就会变得火辣辣的。

【谷歌译文】我是气象员, 当店员不友好或脸闷时, 它会发出咔嗒声。

“拉気屋” 译为 “喜怒无常的人、没准脾气的人” (像天气那般不可猜 测)。以上可见, 机器翻译容易直译。此外, “カチンとくる” 是日语惯用语, 表示 “生气”。谷歌译文实则是把 “カチン” 和 “くる” 分开翻译, 于是把 “カチン”翻译成拟声词 “咔嗒”。

\section{4. 机器翻译与人工翻译的差异}

虽然机器翻译与人工翻译都是为了精确地把一种语言转换为另一种语 言, 而且, 对简短句的翻译有时可以完全一样, 但差异仍非常明显。

第一、速度。由于机器翻译拥有计算机系统, 运算时间极快, 且一次性 处理的信息量能比人工多。在使用 APP 翻译的时候, 不到 2 秒便能得到译文。 此外, 据说语音识别翻译的速度为每秒钟 400 词, 是同声传译的 200 倍。可 见二者速度之差。

第二、成本。翻译 APP 一般而言是免费的。不过, 也有的 APP 设有收 费的人工翻译服务。人工翻译的情况下, 笔译一般按字收费, 每千字几百人 民币不等; 口译多按时收费, 一天几千人民币甚至上万。显然, 机器翻译的 使用成本要更低。

第三、准确性。机器翻译容易出现直译。语言具有主观性, 它有惯用语、 浐语、双关语等表达。即使语言学家总结了语言的规律, 这些规律也不能套 用于所有情况。加上国家长远历史的发展, 使得语言背后有着深厚的文化背 景。很多时候, 翻译不仅是文字的转换, 更是心与心的交流。机器翻译缺乏 人类思维, 无法真正理解各种语境, 只能依靠数据进行翻译。且由于数据不 全, 不能翻译到位, 有时反而容易出现错误, 需要人工校对。而人工翻译在 这方面毫无疑问要出色得多。

第四、信息储存。机器翻译可以储存大量信息且不会产生遗忘。比如在 翻译专有名词上, 拥有专业名词语料库的机器翻译要比人工翻译效率高。记 忆专有名词本身难度就大, 再加上人脑会产生遗忘, 使得人工翻译要逊色一 筹。

第五、持续工作时间。在人工翻译的过程中, 译员需要集中注意力, 长 时间地输入输出信息, 非常消耗脑力。因而会产生疲忞, 可能出现漏译、误 译等情况, 从而影响效率。而机器翻译不具主观性, 可以反复机械地操作, 
恰好弥补了人工翻译的主观性、生理性的缺点。

\section{5. 机器翻译与人工翻译的关系}

\section{1. “错开”与 “合作”}

机器翻译与人工翻译各有所长。与其讨论 “人工智能是否会取代人类” 这样绝对的话题, 不如思考如何结合二者, 即根据不同情况, 二者互相 “错 开” 或 “合作”。在翻译普通资料、旅游等一些轻松的情况下, 机器翻译能 高效率地解决问题; 在翻译文件需要高准确率、重要谈话等情况下，人工翻 译能更稳重地完成任务。当然, 两者配合则事半功倍。注重机器翻译的便利 性, 发挥人工翻译的主体性, 不仅能取长补短, 还能提高劳动效率。

\section{2. 共同进步}

科技进步是时代的潮流。完善机器翻译语料库, 并利用机器翻译系统中 的神经网络, 让机器翻译接近人类思维, 以此提高翻译的准确性、专业性。 与此同时, 译员也应提高自己的专业水平, 最好是能学习计算机专业知识, 将自己的领域和机器翻译结合起来，未来一定会备受青睐。

\section{6. 结语}

综上所述, 机器翻译与人工翻译各有利弊。机器翻译以人工翻译为基础, 人工翻译也难以舍弃机器翻译带来的便利性。人工智能不可能完全代替人类, 未来的机器翻译也不可能完全取代人工翻译。如果有朝一日变为现实, 那么 不止是翻译行业, 整个社会的各行各业都将被人工智能所取代[4]。科技与人 类相配合是未来的必然趋势。

由于笔者处于本科阶段, 知识尚浅, 本文研究存在一定的片面性。文中 虽提到了两种重要的机器翻译方式, 但至今为止机器翻译方式并不只有两种, 其优劣势及运作方式分析的不够完整清晰, 需要再研究清楚。此外, 所举的 翻译例子有限, 没有很好地系统分析人工译文和机器译文在语法、用词上的 差异。这两方面仍需进一步研究。

\section{Conflicts of Interest}

The authors declare no conflicts of interest.

\section{References}

[1] 高宁. 汉日翻译教程 修订版 [M]. 上海: 上海外语教育出版社, 2013.

[2] 吴㑆. 汉日翻译技巧与实践 $[\mathrm{M}]$. 上海: 上海外语教育出版社, 2006.

[3] 高宁. 日汉翻译教程 [M]. 上海: 上海外语教育出版社, 2008.

[4] 宋仕振. 试论机器翻译与人工翻译的未来关系 [J]. 未来与发展, 2019, 43(2): 25-30. 


\section{Appendix (Abstract and Keywords in Chinese)}

\section{机器翻译与人工翻译对比思考一一以日语翻译类 APP 为例}

摘要: 随着人工智能的发展, 机器翻译也在不断进步。本文将以日语翻 译类 APP 为例, 对机器翻译与人工翻译的对比差异进行分析, 并探讨二者的 关系及对未来的展望。

关键词: 人工智能, 机器翻译, 人工翻译, 对比差异, 关系 